\title{
¿Puede la química hacerte más bella? Una propuesta didáctica desde el modelo de enseñanza para la comprensión
}

\author{
Alexandra Ortiz Huertas \\ dqu706_jortiz@pedagogica.edu.co \\ Jonatan López Castillo \\ dqu695_jlopez@pedagogica.edu.co

\section{Resumen} \\ El presente trabajo tiene como objetivo presentar a la comunidad educativa \\ los resultados obtenidos a partir del diseño de la unidad didáctica ¿Puede la \\ química hacerte más bella?, la cual fue aplicada a estudiantes de grado Once \\ del IED Liceo Femenino La Merced, con la finalidad de mejorar sus habilida- \\ des de pensamiento.
}

\section{Palabras clave}

Habilidades de pensamiento, enseñanza para la compresión, concentración, motivación. 


\section{Problema}

¿La comprensión de la temática de clases de reacciones en Química Orgánica (según su mecanismo) fomenta el desarrollo de las habilidades de pensamiento en las estudiantes de grado Once de la Institución Educativa Distrital Liceo Femenino Mercedes Nariño?

\section{Objetivos}

1. Diseñar y aplicar una propuesta de intervención didáctica para comprender las clases de reacciones químicas orgánicas, para estudiantes de grados Once del IED Liceo Femenino Mercedes Nariño, desde el modelo de enseñanza para la comprensión, con el fin de fomentar el desarrollo de sus habilidades de pensamiento.

2. Presentar a la comunidad académica los resultados y el análisis respectivo de esta experiencia de aula.

\section{Marco Teórico}

La Enseñanza Para la Comprensión -EPC- ha adquirido una importancia determinante en los sistemas educativos y se ha constituido como parte de la agenda olvidada en las reformas educativas. Por estas razones se debe prestar atención prioritaria a este modelo, puesto que existe consenso sobre su eficacia escolar (Duarte, 2001).

Este modelo fue creado en 1990 por un grupo de la Escuela de Graduados de Harvard, en el que se destacan H. Gardner, D. Perkins y V. Perrone, con el fin de fomentar en los educandos un pensamiento crítico y una capacidad de sortear la complejidad de los fenómenos, planteando alternativas de solución a diferentes problemas. Estos investigadores se apoyan en la convicción de que las escuelas deben comprometer a los estudiantes con su traba- jo intelectual, haciendo de la comprensión su pieza central (Gardner, 2001).

Se define la comprensión como un proceso interactivo, en el cual el sujeto ha de construir una representación ordenada y coherente del aprendizaje, relacionada con sus conocimientos previos, con el objetivo de reflexionarlos y transcenderlos más allá de algunas imágenes mentales (Stone, 2003).

La EPC propone los siguientes procesos, como aspectos claves en el aprendizaje:

\section{Tópicos generativos}

Son cuestiones, conceptos o ideas que proporcionan hondura, significación y conexiones variadas para apoyar el desarrollo de compresiones profundas en el estudiante.

\section{Metas de comprensión}

Son relaciones o preguntas que determinan el itinerario de trabajo a partir del tópico generativo. Estas pueden clasificarse como hilos conductores (metas de compresión amplias para un curso) y metas de comprensión (objetivos específicos, propios para cada unidad temática).

\section{Desempeño de comprensión}

Son las formas en que se expresan las capacidades e inclinaciones para usar el conocimiento adquirido.

Una de las mayores preocupaciones del modelo consiste en investigar la forma de averiguar lo que comprenden los educandos; por ende, se presenta la necesidad de emplear una evaluación diagnóstica continua, caracterizada por su componente descriptivo. Así, el educador va registrando los avances en la comprensión de acuerdo con los estilos y ritmos de aprendizaje personalizados de los educandos; con ello podrá luego compararlos con las metas de comprensión propuestas para una unidad determinada. 
Desde este enfoque, existe la necesidad de hacer una autoevaluación, co-evaluación y heteroevaluación (Flórez,1999), lo que permite integrar mejor la perspectiva holística de los contenidos investigados y los instrumentos intelectuales. Estos pueden incluir mapas mentales, mentefactos, mapas conceptuales y otras representaciones mentales que permitan la jerarquización del conocimiento y su estructuración en el sujeto (Pedro, 2001) y las operaciones intelectuales construidas en el aula o fuera de ella.

\section{Metodología}

\section{Fase de diagnóstico}

Se realiza una evaluación de las habilidades de pensamiento a través de un instrumento que se presenta en forma de cuestionario, el cual contiene 61 reactivos de tipo Likert que en su conjunto están destinados a reunir, de manera sistemática y organizada, información que permita evaluar los hábitos y habilidades de estudio de las estudiantes de educación media de IED Liceo Femenino la Merced.

El instrumento está organizado en cinco sub-campos que permiten evaluar:

- La utilización y desarrollo de pensamiento crítico.

- Tiempo y lugar de estudio.

- Uso de técnicas de estudio.

- Capacidad de concentración en tareas académicas.

- Motivación.

Para la elaboración de la puntuación, los ítems se puntúan del 1 al 2 para cada columna; por efectos de la concordancia interna del instrumento, en la población solo se puntúa la columna de la facilidad o dificultad para llevar a cabo la actividad que plantea el ítem.

Cada una de las cinco dimensiones está representada por un grupo de afirmaciones, las cuales se encuentran organizadas de la siguiente manera:

\section{Pensamiento crítico}

(reactivos 6, 7, 10, 13, 14, 33, 42, 46, 50, 51, 52, $53,54)$.

\section{Tiempo y lugar de estudio}

(reactivos 3, 4, 5, 24, 27, 29, 31, 40, 41).

\section{Técnicas de estudio}

(reactivos 1, 2, 8, 9, 15, 19, 21, 22, 23, 25, 30, 45, $47,48,58)$.

\section{Concentración}

(reactivos 11, 12, 20, 32, 35, 36, 37, 39, 49 y 60).

\section{Motivación}

(reactivos 16, 17, 18, 26, 28, 38, 43, 44, 56, 59, 61).

Cada conjunto de notas posee una puntuación máxima alcanzada, la cual está determinada por el número de reactivos presentes en cada conjunto. La mayor puntuación por pregunta es de 2; por ende, si la dimensión de pensamiento crítico posee 15 reactivos, el máximo puntaje alcanzado es 30. Con ello se determina un promedio por grado analizado y porcentaje individual por estudiante, para, finalmente, hacer una gráfica que revele una tendencia de nivel de las cinco dimensiones de habilidades de pensamiento. Las gráficas pueden hacerse de manera individual o por grupos de varias estudiantes.

El instrumento se aplica al antes y después de la aplicación de la propuesta didáctica en las estudiantes de grado 1101 y 1104 del IED Liceo Femenino Mercedes Nariño.

\section{Fase de diagnóstico}

La propuesta de intervención tiene como fin el diseño de una unidad didáctica que mejore las habilidades de pensamiento de las estudiantes de grado once del IED Liceo Femenino La Merced. La unidad didáctica bajo este modelo lleva por nombre ¿La Química puede hacerte 
más bella? Esta tiene como base la elaboración de unos tópicos generativos, metas y desempeños de comprensión, de los cuales se desprenderán los criterios necesarios para llevar a cabo una evaluación continua y descriptiva

La unidad está dividida en tres partes, correspondientes a las sesiones de trabajo presencial, trabajo extra-clase y el proyecto final de síntesis.

Las sesiones de trabajo dentro de la unidad están organizadas a partir de 3 momentos:

Tiempo del estudiante

Se plantea una zona o un tiempo específico para el trabajo con las estudiantes, en donde ellas tendrán la oportunidad de realizar actividades lúdicas o académicas, referidas a tiempos de lectura, tiempos de juego, tiempos de preparación para el examen ICFES, tiempo de aprendizaje de algoritmo, tiempo de trabajo en grupo o trabajo individual.

\section{Tiempo del docente}

Teniendo en cuenta la metodología del docente, en este tiempo realiza una orientación de tipo expositivo con respecto del tema a trabajar. En esta exposición se contemplan elementos de tipo interdisciplinar, con el fin de contextualizar el conocimiento y hacerlo más llamativo e interesante para las estudiantes.

\section{Tiempo de juego}

A través de algunas actividades lúdicas relacionadas con el tema, el estudiante despliega sus habilidades mediante la acción. En este tiempo se desea ir realizando una evaluación diagnóstica continua, al revelar las dificultades de las estudiantes o sus capacidades para llevar a cabo la actividad.

Dichas actividades son creadas bajo la perspectiva de aprender haciendo, donde el material entregado constituye la interface entre la acción del estudiante y el conocimiento de la misma.

Las sesiones extra-clase están compuestas por retos semanales para las estudiantes y una preparación a través de preguntas guiadas para presentar la prueba ICFES, como una demanda institucional.

El proyecto final de síntesis consistió en la elaboración de un producto cosmético que contemplaba el diseño de un logo y etiqueta según la normatividad colombiana vigente en cosmetología y un comercial en donde las estudiantes argumentaran las propiedades del cosmético a nivel químico.

\section{Resultados y análisis}

A continuación se presentan dos gráficas que contienen la información de los porcentajes obtenidos para cada ítem evaluado por el instrumento, en los dos momentos importantes de la investigación.

Las columnas de coloración intensa indican los porcentajes obtenidos antes de la aplicación de la propuesta, mientras las columnas de coloración más clara representan los porcentajes obtenidos después de la intervención realizada con la unidad didáctica.

La gráfica 1 corresponde al grado 1104 y la gráfica 2 corresponde al grado 1101 de la mencionada institución educativa.

Se encuentra un incremento significativo en los porcentajes correspondientes a pensamiento crítico, concentración y motivación, mientras que ítems tales como técnicas de estudio y tiempo y lugar de estudio presentan un aumento menor. Sin embargo, se observa un incremento general en todos los ítems luego de la aplicación de la unidad didáctica, una mejoría en las estudiantes de dichos cursos. 
Se observa un incremento promedio del $17 \%$ para cada ítem, lo que justifica la efectividad o la pertinencia del empleo de la propuesta metodológica para desarrollar habilidades de pensamiento crítico.

Dichos resultados presentan concordancia con lo evidenciado en el comportamiento de las estudiantes, quienes se encontraban motivadas en el trabajo de la asignatura, lo que se reflejó en su participación en las actividades de aula, sus propuestas para mejorar el ambiente de la clase, su cumplimiento con las tareas a realizar, la entrega de un trabajo de calidad en referencia a su proyecto final de síntesis; todo ello a pesar de reconocer un rechazo colectivo hacia la asignatura por considerar muy compleja la asimilación de dicho conocimiento.

Si bien las estudiantes enriquecieron su vocabulario en el área de Química y lograron mantener un dominio en las relaciones de sus conceptos, sus explicaciones fueron más complejas y sus cuestionamientos con respecto a la realidad iban más enfocados a ser interpretados desde una perspectiva en Química, en la medida en que se desarrollaba el tópico generativo.

Además se encuentra una coherencia entre los resultados suministrados por las gráficas y los datos obtenidos a partir de los trabajos realizados en el aula, los cuales fueron evaluados a través del uso de las matrices diseñadas previamente, caracterizadas por su carácter público y unánime.

En ellas se evidencian específicamente habilidades de pensamiento crítico, a través de actividades diseñadas con el objetivo de mejorar la esfera motivacional y lograr la asimilación de un conocimiento en reacciones químicas orgánicas.

A partir de las acciones de las estudiantes y de sus manifestaciones en el aula, se encontró que a partir de la unidad didáctica:

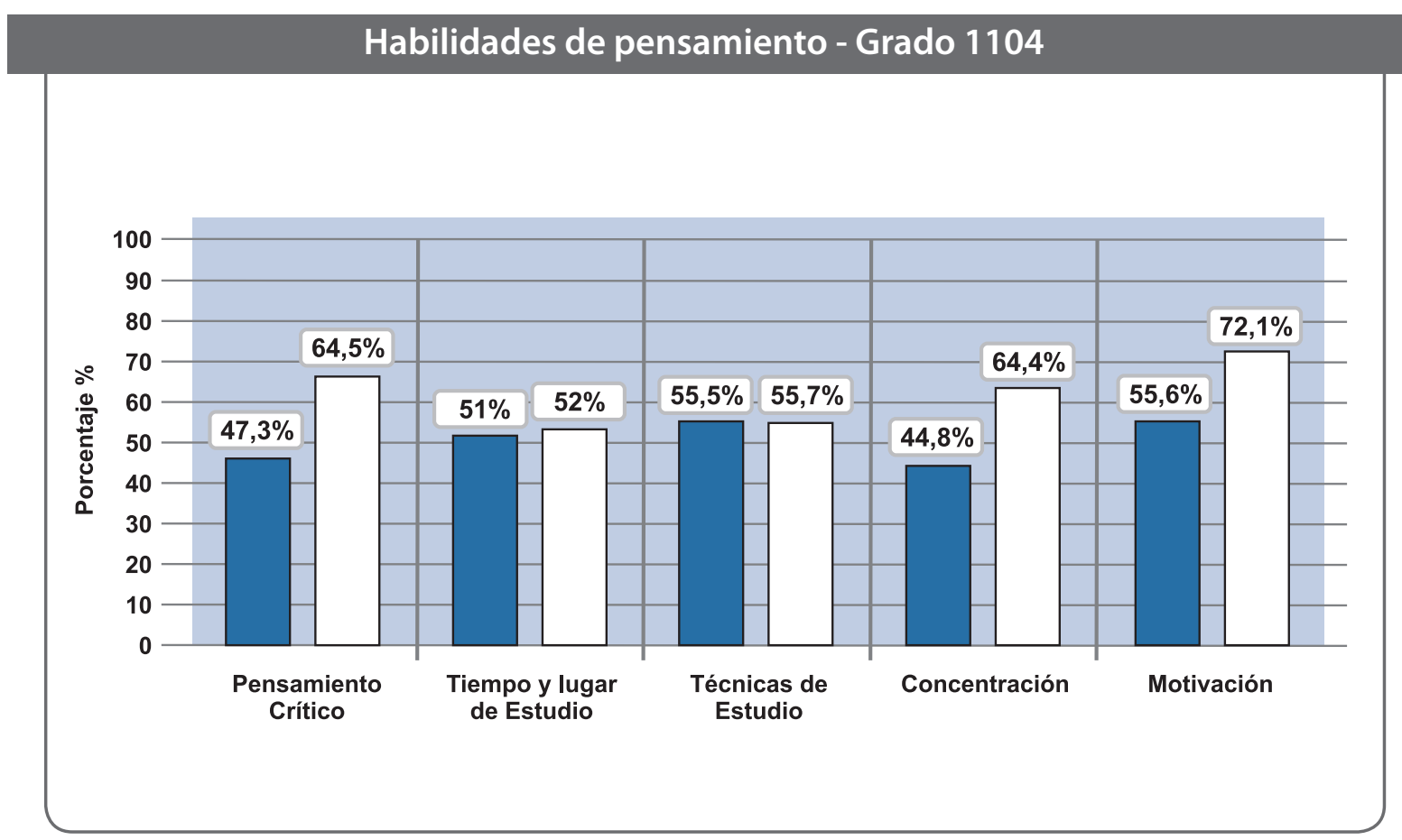

Gráfica 1 Evaluación de las habilidades de pensamiento de las estudiantes de grado 1104. 


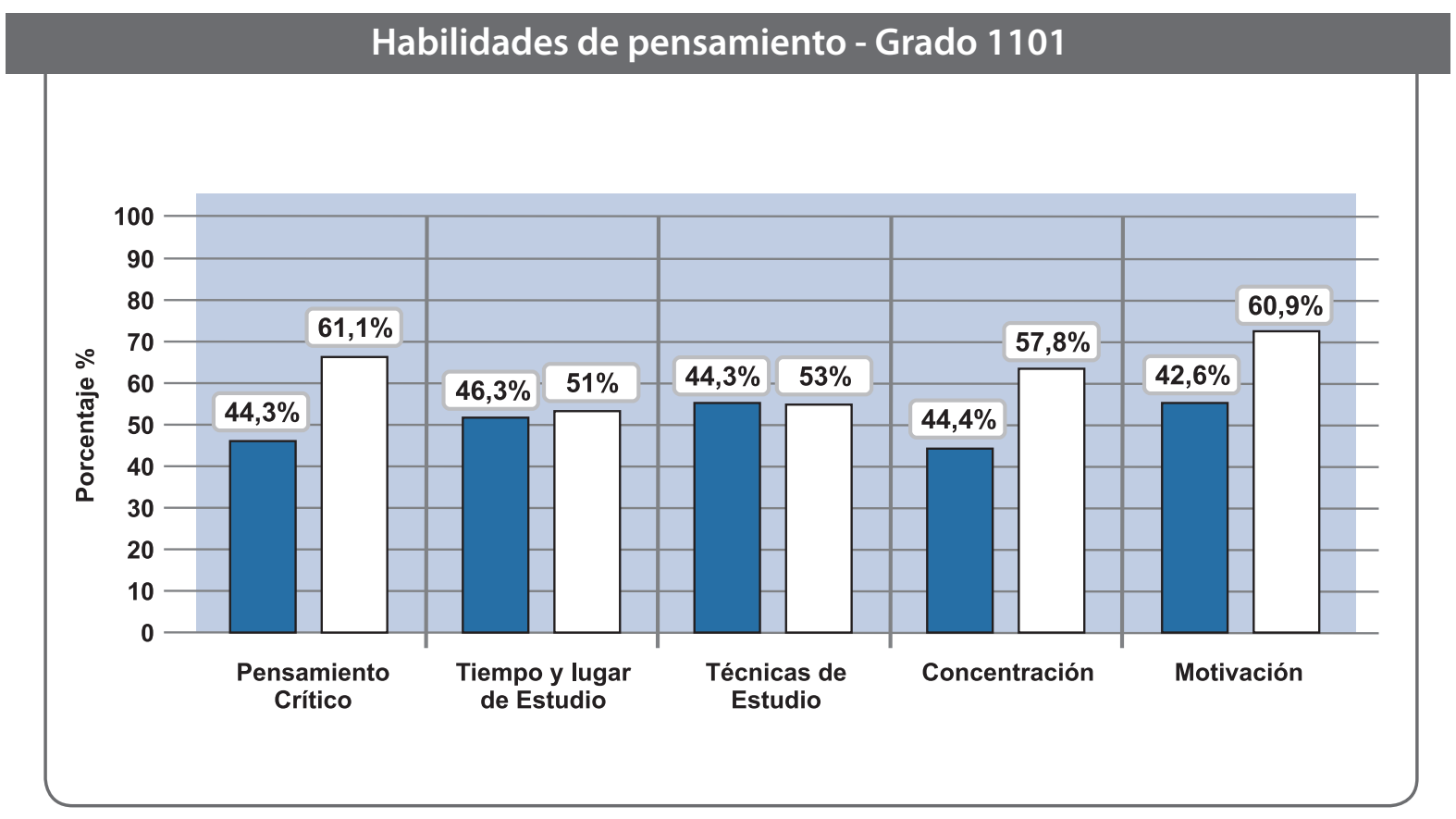

Gráfica 2 _Evaluación de las habilidades de pensamiento de las estudiantes de grado 1101.

Las estudiantes adquieren una perspectiva más amplia de la evaluación y su carácter retroalimentador, puesto que comprenden que todo su trabajo es valorado y no solo se limita a la presentación de una prueba escrita u oral.

Se genera un clima de aula menos tensionante y más participativo, en el que las estudiantes pueden detectar sus propios errores y corregirlos.

Se propician espacios para el trabajo autónomo y creativo de las estudiantes.

Se crean momentos para compartir experiencias cotidianas y su comprensión a través de la relación que tienen con la química.

Si se tiene en cuenta un análisis de la dinámica en el aula, se encuentra que las dos gráficas reflejarían la evaluación de los cinco ítems en dos realidades educativas diferentes. Cada una de ellas está mediada por concepciones de ciencia, evaluación y educación distintas, las cuales implican un diseño metodológico particular y unos resultados propios de las capacidades de las estudiantes.
A partir de una perspectiva empiro-inductivista se tiene una metodología de enseñanza que favorece las capacidades de memorización y estimula una operacionalización de la evaluación, como una práctica reduccionista, estereotipada y facilista que no hace uso de su poder de retroalimentación.

En cambio, desde un enfoque constructivista a partir del modelo de enseñanza para la comprensión, se crean espacios donde el estudiante se enfrenta a nuevos retos de aprendizaje, favoreciendo espacios de reflexión para compartir experiencias, haciendo uso del error para buscar mejoras y con ello, aunque se emplee más tiempo, se logran establecer compromisos de aprendizaje entre los agentes que componen el proceso.

A través de los resultados obtenidos se da una interpretación a favor de una relación entre los cambios metodológicos en la enseñanza de la Química y el desarrollo de habilidades de pensamiento crítico, comprendido 
en capacidad de síntesis, análisis, proposición y reflexión.

Sin embargo, no se puede especificar una relación directa, puesto que se observaría un incremento proporcional en todos los ítems y, de acuerdo con esto, las estudiantes habrían reflejado los mismos cambios significativos a través de los diferentes cambios metodológicos a los que se han visto expuestas a lo largo de su recorrido en la escuela en esta área de conocimiento.

Según el docente titular, los cambios metodológicos no constituían una causa directa para mejorar el trabajo de las estudiantes, ni su capacidad para resolver problemas, ni sus perspectivas negativas con respecto al trabajo en Química; esto significaba que la propuesta abordaba una esfera específica que permitía el desarrollo de las habilidades de pensamiento (la postura inicial desde la cual se ha articulado este trabajo).

De esta forma se halla que, a partir de diseñar unidades cuyos contenidos sean abordados teniendo en cuenta los intereses y realidades del estudiante, se favorece el trabajo y el alcance de los logros esperados, incrementando la participación, incitando a sus pasiones intelectuales y guiando sus acciones hacia situaciones complejas en donde se refleje y se construya su aprendizaje.

Esta nueva connotación enriquece la investigación en la medida en que transforma la concepción del problema abordado, ya que este análisis concuerda con investigaciones en las que se ha especificado la importancia que cobra la disposición de los estudiantes en el desarrollo de sus habilidades y su concentración, puesto que las estudiantes se concentrarán en aquello que consideren interesante e importante y, con ello, invertirán tiempo y esfuerzo en indagar y aprender más sobre esta área del conocimiento.

\section{Conclusiones}

No existe una relación directamente proporcional entre el desarrollo de habilidades de pensamiento y la enseñanza de la Química expresada en la metodología docente. Sin embargo, existe una relación entre la evolución de dicho componente cognitivo y una planificación metodológica basada en las disposiciones, pasiones e intereses de las estudiantes, lo que indica una relación entre motivación y desarrollo de habilidades de pensamiento.

Para que exista el desarrollo de un componente conductual hacia el pensamiento crítico, desde el diseño metodológico se deben planificar actividades que motiven a las estudiantes a un trabajo voluntario, además de espacios de reflexión en donde ellas puedan articular su conocimiento a su práctica de vida.

\section{Bibliografía}

Blythe, T. (1999). La enseñanza para la comprensión. Guía para el docente. Buenos Aires: Editorial Paidós.

Caminolli, A., Davini, M. C., Edelstein, G., Litwin, E., Souto, M. y Barco, S. (1998). Corrientes didácticas contemporáneas. Cuestiones de Educación. Buenos Aires: Editorial Paidós.

Ege, S. (1998). Química Orgánica Estructura y Reactividad. Barcelona: Editorial Reverté.

Fessenden, J.R., Fessenden S. J. (1983). Química Orgánica. México: Grupo Editorial Iberoamericana.

Flórez, R. (1999). Evaluación pedagógica y cognición. Bogotá: McGrawHill.

Gardner, H. (2001). Inteligencias Múltiples. México: Editorial Reverté.

Osma, Y. (2001). Las organizaciones escolares integrales: enseñanza para la comprensión. Bogotá: Editorial Magisterio. 